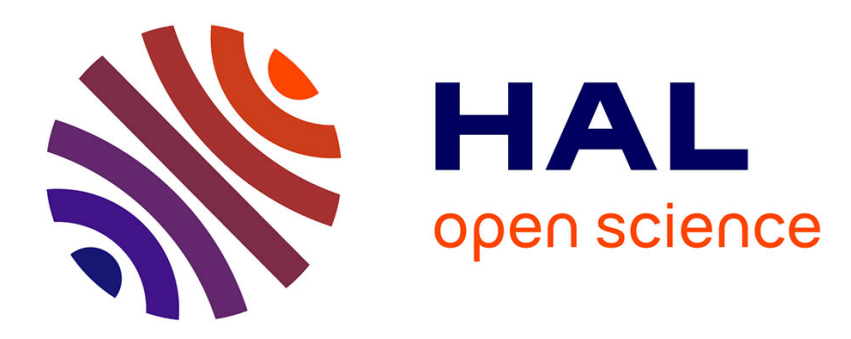

\title{
Des liens humains (toucher, contagion, sympathie) Philippe Roussin
}

\section{To cite this version:}

Philippe Roussin. Des liens humains (toucher, contagion, sympathie). Communications, 1998, La contagion, 66, pp.121-146. hal-03164336

\section{HAL Id: hal-03164336 \\ https://hal.science/hal-03164336}

Submitted on 11 Mar 2021

HAL is a multi-disciplinary open access archive for the deposit and dissemination of scientific research documents, whether they are published or not. The documents may come from teaching and research institutions in France or abroad, or from public or private research centers.
L'archive ouverte pluridisciplinaire HAL, est destinée au dépôt et à la diffusion de documents scientifiques de niveau recherche, publiés ou non, émanant des établissements d'enseignement et de recherche français ou étrangers, des laboratoires publics ou privés. 


\section{Des liens humains (toucher, contagion, sympathie)}

Philippe Roussin

\section{Citer ce document / Cite this document :}

Roussin Philippe. Des liens humains (toucher, contagion, sympathie). In: Communications, 66, 1998. La contagion. pp. 121146;

doi : https://doi.org/10.3406/comm.1998.2007

https://www.persee.fr/doc/comm_0588-8018_1998_num_66_1_2007

Fichier pdf généré le 10/05/2018 


\section{Philippe Roussin \\ Des liens humains (toucher, contagion, sympathie)}

L'usage de la communication dans une société de tradition chrétienne, où les liens sociaux sont fondés en référence à une transcendance et à une Providence; l'évolution de sens et d'usage du verbe toucher, introduit par la rhétorique sacrée d'après le concile de Trente dans l'édifice rhétorique et venant le désorganiser; la mise en question du mode de pensée et de communication de la rhétorique par la critique rationaliste, sous l'espèce de la contagion; le passage, au tournant du XVIII' siècle, pour le pire et le meilleur, du verbe toucher de la rhétorique à l'esthétique ; enfin, l'anti-providentialisme de la philosophie anglaise, de Hume notamment, qui permet de penser le contact et le lien entre les hommes non plus en termes d'influence et de contagion mais de sympathie: telles sont les principales questions qui organisent les remarques qui suivent. Trois termes tissent ainsi le fil de l'analyse : toucher, contagion et sympathie'.

\section{Toucher.}

Dans le courant du XVII siècle, toucher apparaît dans les traductions des auteurs antiques et néo-latins comme dans les traités de rhétorique rédigés dans la langue nationale, à côté de persuader, d'émouvoir et de fléchir et vient désorganiser la répartition de leurs usages respectifs. Un bon exemple en est donné par l'évolution des traductions du De doctrina christiana d'Augustin, notamment du livre consacré à la rhétorique. G. Collet choisit encore, en 1636, de traduire flectere par émouvoir ou fléchir : "l'homme éloquent doit parler de telle sorte qu'il enseigne, qu'il délecte et qu'il émeuve »; "il faut donc que le docteur ecclésiastique [...] n'enseigne pas seulement [...] mais aussi qu'il fléchisse ». Cognard, en 1701, optera pour toucher. I.e texte de 1636 indique qu'il convient de " délecter » lauditeur, celui de 1701 que celui-ci est " touché quand on 


\section{Philippe Roussin}

lui parle avec agrément ». Cognard, lecteur du traité $\mathrm{Du}$ sublime de Longin, après Boileau, affirme qu'on peut «toucher en posant de grandes questions en style sublime [ut flectat, magne dicenter dicere] " : " un moyen pour amener l'auditeur à donner son assentiment, c'est de le toucher [flectere] par la puissance de l'éloquence".

Si l'on veut situer le lieu et les causes de l'émergence du mot, dont l'apparition sera l'occasion d'une réorganisation interne de la rhétorique et qui influencera le vocabulaire de l'esthétique sentimentale au XV'III" siècle, c'est du côté de la rhétorique chrétienne qu'il faut se tourner. A l'origine de la prééminence conquise par le verbe et de ce qu'il circonscrit, il y a le courant post-tridentin réuni autour du réformateur des grands séminaires, Charles Borromée, les traités de rhétorique sacrée tels ceux de Valerio (De rhetorica ecclesiastica ad clericos, 1574) et de Botero (De praedicatore verbi Dei, 1585), composés par ordre du prélat pour être enseignés aux jeunes clercs, ou celui du spirituel espagnol Louis de Grenade (Ecclesiasticae rhetoricae, 1578). L'orateur chrétien, écrit ce dernier dans sa Rhétorique de l'Église, doit «enseigner, plaire et toucher » : «il est de nécessité d'enseigner et de faire comprendre, ce que l'on veut persuader ; de plaire en l'enseignant, afin de persuader avec douceur; de toucher et d'émouvoir les cœurs, afin qu'on s'y rende et qu'on se laisse vaincre $" ~ "$. Valerio consacre deux chapitres de sa Rhetorica ecclesiastica à définir ce qu'est toucher ("Quid sit commotio ? ») et aux moyens propres pour y parvenir ( Qua ratione auditorum animi possint commovere ») : " le premier devoir du prédicateur est d'instruire, il peut chercher à plaire $[\ldots]$ mais quand il touche et persuade, quand il triomphe des cœurs par la force de ses discours, c'est alors qu'il est éloquent ${ }^{3}{ }^{\prime}$.

Pour cette rhétorique sacrée, il s'agit moins de persuader de la justesse d'une cause ou du bien-fondé de la vérité que de toucher pour ramener à la vérité. Persuader et savoir ce qui est bien'ne suffisent pas à le faire réaliser par l'action : "il suffit à un Dialecticien, qui veut persuader une chose dont on doute, de l'enseigner simplement, c'est-à-dire d'en montrer la vérité et d'en convaincre ses Auditeurs par la force des preuves et des arguments »; mais le devoir de l'orateur chrétien étant « de les exciter à faire ce qu'il leur persuade ", "il doit aussi faire en sorte par la beauté de ses discours, et par la variété de son style, qu'ils se plaisent à les entendre, qu'ils en soient touchés, et qu'ils se portent à les pratiquer ", écrit Louis de Grenade ${ }^{+}$. Et Valerio : "Quoiqu”il ne paraisse pas nécessaire de prouver à des chrétiens la nécessité où ils sont de remplir les devoirs du christianisme parce que leur propre intérêt les y engage et que leur salut en dépend, il faut cependant exciter leur foi, l'animer dans ce combat continu qu'ils doivent soutenir contre les ennemis du salut."

L'orateur chrétien persuade en touchant et ce qui ne touche pas est 
déclaré sans valeur au regard du but que la rhétorique sacrée se donne. Toucher s'impose, dès lors, comme une des fins de la prédication : "que la vérité nous soit connue, que la vérité soit écoutée avec plaisir, que la vérité nous touche [ut veritas pateat, ut veritas placeat, ut veritas moveat] »; lorsque le terme se trouvera consacré, Rollin pourra affirmer que "Saint Augustin, en suivant le plan que Cicéron nous a tracé des devoirs de l'orateur, dit qu'ils consistent à instruire, à plaire, à toucher." ${ }^{.3}$. Parfois, ainsi chez François de Sales, enseigner et émouvoir ("éclaircir l'entendement des auditeurs et échauffer les volontés ») suffisent à fournir la définition de l'éloquence chrétienne, excluant ainsi de la rhétorique sa troisième fonction (plaire) ${ }^{\circ}$. Citant Louis de Grenade et résumant une tradition, l'auteur de l'article «Mouvements oratoires » du volume consacré à la rhétorique sacrée dans l'Encyclopédie Migne ira, au siècle dernier, jusqu'à faire de toucher le premier but de l'éloquence chrétienne, indiquant que c'est là ce qui la spécifie et la distingue de l'ensemble des différents genres de l'éloquence profane : "la principale fonction du prédicateur de l'Évangile est plutôt de toucher les coeurs et de remuer les affections des auditeurs que d'éclairer leurs esprits, parce que les hommes pèchent bien plus par la corruption du cœur, que par l'ignorance de la vérité ».

Toucher et ce que le terme circonscrit sont clairement reconnus comme constituant le domaine propre de la rhétorique de la chaire : "L'éloquence, que l'on peut justement appeler la Science de la parole, est l'Art de persuader l'esprit, et de toucher le cour, indique l'abbé de Bretteville. Cette définition convient également à l'Éloquence de la Chaire, et à l'Éloquence du Barreau. Il y a néanmoins cette différence, que l'Éloquence de la Chaire tend principalement à toucher le cœur, au lieu que l'Éloquence du Barreau a pour fin particulière de persuader l'esprit ? ? B. Lamy en fournit une preuve a contrario, lorsqu'il justifie l'absence de tout traitement de la prédication dans sa Rhétorique : "ce qui fait la différence des prédicateurs qui instruisent les peuples et des avocats, c'est que l'orateur a pour auditeurs des juges qui ne se veulent point persuadés que par la force d'un raisonnement exact. On est convaincu de ce que dit le Prédicateur : on ne le va entendre que pour être touché de quelque sentiment de dévotion ${ }^{8} »$.

Toucher, faut-il le souligner, n'est pas le terme précisément attendu pour traduire persuadere, movere ou flectere. Le verbe et le domaine qu'il circonscrit appartiennent, en fait, à l'expérience de la communication directe avec Dieu, telle que l'ont décrite les auteurs mystiques puis, à partir du XVII" siècle, la théologie mystique. Toucher traduit le tactus spiritualis, le toucher de l'âme, la survenue de l'expérience spirituelle, le 


\section{Philippe Roussin}

tactus dei, la touche divine qui se communique à l'âme au plus haut de l'expérience mystique, le gherinen des mystiques rhéno-flamands et de Van Ruysbroeck, le toque de Jean de la Croix et des mystiques espagnols". Dans le cheminement mystique qui conduit de la purification à l'illumination et à la perfection, toucher appartient à l'étape de l'union mystique, décrite sous les traits d'un banquet ou de noces spirituelles, au cours de laquelle le sujet fait l'expérience de la présence intérieure de Dieu en lui : « ces communications que Dieu fait par lui-même, écrit Jean de la Croix dans La Montée du Carmel, sont souveraines et totalement divines. [...] Ces communications constituent le plus haut degré de l'oraison [...]. Ce sont ces touches que l'épouse sollicitait dans le Cantique des cantiques en disant : "Qu'il me baise d'un baiser de sa bouche" (Ct 1,1). C'est une faveur si intime que cette touche de la Divinité, et l'âme y aspire avec tant d'ardeur qu'elle la met au-dessus de toutes les autres faveurs que Dieu lui accorde ${ }^{10}$ ». Les touches résultent d'étapes actives et passives : l'expérience s'opère par un effort personnel d'amour ou, au contraire, découle de la touche divine par laquelle Dieu fait sentir sa présence ou son action, le perfectionnement intérieur passant par tous les stades et les expériences décrits dans les relations des auteurs mystiques.

Dans cet état où l'âme a été élevée à la dignité d'épouse du Christ et savoure son contact, l'Esprit-Saint se communique directement "par le moyen de touches divines qui produisent un accroissement ou de nouvelles modalités ou de nouveaux degrés de grâce " ", indique un autre auteur espagnol ; cette touche, poursuit-il, "n'est autre chose qu'un sentiment très intense, très profond et très puissant de la présence de Dieu en l'âme, sentiment causé par le toucher de Dieu qui, par la force de sa présence et l'union de sa substance à la substance de l'âme, fait éprouver son contact et laisse l'âme pleine des heureux effets de ce toucher, de ce passage divin [...] dans l'intelligence, c'est une nouvelle manifestation, une nouvelle connaissance si claire et si simple de la vérité éternelle, de la souveraine Autorité, de la Toute-Puissance, que les mots manquent pour en dire la perfection".

Jean de Jésus Maria, dans sa Théologie mystique, qui repose sur les écrits de sainte Thérèse, décrit, après les ravissements, les extases, les cris de joie, les gémissements, les tremblements des membres, les sauts, et autres "impulsions véhémentes» qui précèdent le toucher du SaintEsprit, la passivité de ce dernier état : "cet occulte touchement de Dieu [...] se fait lorsque sans qu'il ait précédé aucun acte d'entendement [...] le Saint-Esprit touche et enflamme du feu de son amour le sommet de l'affection [...], le faîte de la volonté, ou la portion correspondante à la partie supérieure de l'entendement [...] quand la volonté est touchée de la sorte, elle pâtit les choses divines, c'est-à-dire, qu'elle est mue, ou éprise 


\section{Des liens humains (toucher: contagion. sympathie)}

d'une façon divine, d'autant que par ce touchement divin, elle semble recevoir l'impression d'une certaine impulsion ou d'un certain mouvement pour la porter vers Dieu comme vers son centre ${ }^{1 \cdot 2} \gg$.

Au plus fort de la réaction anti-mystique du Xill siècle, avant que celle-ci ne culmine dans la controverse quiétiste (lorsque Bossuet, dans son Instruction sur les états d'oraison, qualifie d'exorbitantes les expressions des auteurs mystiques, Van Ruysbroeck et Tauler notamment, et critique la manière dont ils enflent le style pour donner une idée de leurs transports), une part du vocabulaire de l'expérience mystique de la communication avec la divinité se trouve ainsi, avec toucher, passer dans le vocabulaire de la communication institutionnelle et de la prédication. C'est à cette communication entre Dieu et l'âme, via la méditation jésuite et l'humanisme dévot, que réfère le toucher qui apparaît alors dans la rhétorique chrétienne.

Suivons la façon dont les traités composent cette expérience individuelle de la communication divine avec les règles de l'éloquence profane. La prédication tire sa force d'une parole intérieure et de sa relation directe avec la puissance même de Dieu : plus la communication avec Dieu est intérieure et pure, écrit Jean de la Croix, plus abondamment Dieu se communique. En d'autres termes, pour toucher son auditoire, l'orateur chrétien devra lui-même avoir été touché. Le prédicateur parlera " du fond du cour suivant les mouvements que l'Esprit de Dieu y met pour toucher les pécheurs ", indique Louis de Grenade : "comment l'Orateur chrétien pourrait-il toucher et remuer les cœurs et les affections de ses auditeurs, poursuit l'auteur, s'il est lui-même sans émotion? Les paroles qui sortent d'un cœur froid, comme dit Saint Grégoire, sont fort peu propres à allumer le feu d'aucun désir des choses du ciel dans ceux qui les entendent. Une chose qui ne brûle pas en elle-même, n'en peut enflammer une autre ${ }^{13}$. Ici réside la différence essentielle entre le prédicateur chrétien et l'orateur profane: "s'il veut être utile au peuple et ne pas tomber lui-même dans la vaine joie et dans la présomption, rappelle Jean de la Croix, il doit se souvenir que sa fonction est plus spirituelle qu'oratoire. Elle s'exerce, il est vrai, par la parole extérieure, mais c'est de l'esprit intérieur qu'elle tire toute sa force et toute son efficacité. En conséquence, si haute que soit la doctrine annoncée, si brillante qu'en soit la rhétorique, si soigné qu'en puisse être le style, le fruit est ordinairement proportionné à l'esprit intérieur du prédicateur. La parole de Dieu, je l'avoue, est efficace par elle-même [...] le feu [...] ne brûle point lorsque la matière inflammable n'a pas la disposition voulue ${ }^{1+}$ ».

Les traités indiquent que l'orateur chrétien se livrera à la prière avant de monter en chaire: « $\mathrm{S}$. Augustin veut que l'orateur chrétien compte 


\section{Philippe Roussin}

beaucoup plus sur la prière que sur ses talents, et qu'avant de parler aux hommes il s'adresse à Dieu, qui peut seul nous inspirer et ce qu'il faut dire et la manière dont il faut le dire ${ }^{1.5}$. » Afin de " se pourvoir du secours de cette efficace des vertus que Dieu a coutume de communiquer à ceux qu'il a choisis pour ministres », note le jésuite G. Mazarini, il se livrera à l'oraison mentale ou à l'oraison vocale : « comme le Saint-Esprit peut seul toucher les coeurs, les pénétrer de sa crainte et de son amour, il faut, observe Valerio, l'invoquer dans nos prières et dans nos sacrifices, pour qu'il nous pénètre les premiers des vérités qu'il annonce par notre bouche ». Et Louis de Grenade : "C'est lui qui embrase les coeurs des Prédicateurs du feu de la Charité : qui les fait brûler d'une ardente soif du salut de leurs frères; qui excite en eux une tristesse et une douleur extrêmes du malheureux état des âmes qui se perdent. » Fénelon explique ainsi la chose dans De la parole intérieure: "A quoi servirait la parole extérieure des pasteurs, et même de l'Écriture, s'il n'y avait une parole intérieure du Saint-Esprit même, qui donne à l'autre toute son efficace ? [...] Ce n'est donc pas seulement la loi extérieure de l'Évangile que Dieu nous montre intérieurement par la lumière de la raison et de la foi ; c'est son esprit qui parle, qui nous touche, qui opère en nous, et qui nous anime. » L'orateur chrétien s'en remettra également à la lecture des Écritures, "pleines de mille figures touchantes» (Fénelon, Dialogue sur l'éloquence en général et sur celle de la chaire en particulier) : " quand le prédicateur s'en est rempli l'esprit, écrit Rapin, il répand dans tout ce qu'il dit cette onction divine, qui est le véritable caractère de la parole de Dieu : et il pénètre les cours par la force de son discours, comme par autant de rayons, pour éclairer l'esprit de ses auditeurs, et comme par autant de flammes pour échauffer leurs cœurs ${ }^{16} \%$. Tandis que la rhétorique profane persuade l'esprit, c'est toujours le cœur, habité par Dieu ou qu'il s'agit d'ouvrir à sa présence, que la rhétorique sacrée s'efforce d'atteindre. Cet appel à la persuasion du cour constitue pour Antoine Arnauld la tâche essentielle de l'éloquence : "c'est principalement sur le cour que l'éloquence chrétienne doit agir »(Réflexions sur l'éloquence des prédicateurs, 1695). Pour Rapin, "la vraie éloquence va au cour, elle touche l'âme et se fait sentir à elle. La fausse n'est qu'un son de paroles qui flatte l'oreille ». L'éloquence qui ne va pas jusqu'au cour plaît, elle ne touche pas.

"Si le coeur est touché, il fournira à celui qui parle la manière de toucher les auditeurs ", alors la langue sera l' "interprète du cœur », veut croire Crevier ${ }^{17}$. Néanmoins, la question se pose : comment toucher le cœur? Les auteurs ont tous conscience de la difficulté. La pénétration des coeurs, la connaissance des secrets, des qualités morales ou immorales 
relèvent du don ou de la grâce. Bretteville rappelle que « la clé de ce cour est réservée au Grand Ouvrier qui l'a formé : lui seul peut l'ouvrir quand il lui plaît, lui seul peut en démêler les mouvements les plus secrets». Rollin le répète, après bien d'autres : "il n'y a que la grâce toute-puissante de Jésus-Christ qui soit capable de toucher ainsi les cœurs, et d'y faire des changements aussi merveilleux. Penser autrement et attendre en quelque degré que ce soit l'efficace de la parole ou des grâces du discours, ou de la solidité des raisons, ou de la force des mouvements, ce serait, selon le langage de s. Paul, anéantir la croix de Jésus-Christ, et lui dérober l'honneur de la conversion du monde pour l'attribuer à la sagesse humaine».

Être touché ne garantit pas que le prédicateur parvienne lui-même à toucher. C'est pourquoi l'orateur chrétien devra "connaître par quelles voies on s'insinue dans l'esprit des hommes et par quels ressorts on parvient à les émouvoir. L'étude du coeur humain est une nécessité indispensable pour lui : et cette étude est une grande partie de la morale» (Crevier, op. cit., p. 6). Bretteville va jusqu'à parler d'une science du coeur; il entend ainsi l'art d'exciter et de rectifier les passions. Mais la question, ici, est surtout celle de l'articulation entre la parole intérieure, reçue, et la parole extérieure, adressée. Quoi que les auteurs en aient "une mission toute divine va-t-elle donc se borner aux artifices d'un rhéteur? » se demandera plus tard Maury -, la prédication devra se mettre à l'école de la rhétorique : "en étant bien persuadé qu'en vain il parlera aux oreilles, si Dieu ne parle aux cours", l'orateur chrétien ne se privera pas de mettre en usage, dit Rollin, "tous les moyens, tous les secours que lui fournit la rhétorique ».

Le moyen de toucher, dans les traités de rhétorique sacrée de l'époque, consistera à faire appel aux passions. Diverses raisons sont avancées. D'abord, la nature sensible de l'homme: Crevier rappelle que "ceux qui nous écoutent ont une raison", mais " aussi des sens; et ce n'est même qu'en parlant à leurs sens que nous pouvons éclairer leur raison »; Bretteville indique que "si l'homme ne se conduisait que par les lumières de son esprit, et s'il ne suivait que la raison pour guide, l'Orateur ne serait pas obligé de se servir de la voix de la Passion pour persuader l'esprit ». Mais c'est' essentiellement une révision importante dans la manière de considérer les passions qui permet l'utilisation que la rhétorique sacrée va en faire. La rhétorique de la Contre-Réforme, qui développe une rhétorique du cœur, réhabilite les passions, moyen de toucher le cœur, à la fois contre une pensée théologique qui les tenait pour un effet du péché originel et contre le néo-stoïcisme du Xv" siècle. Valerio ouvre ainsi son chapitre "Qu'est-ce que toucher»: «les Stoïciens qui veulent rendre 


\section{Philippe Roussin}

l'homme insensible avilissent l'humanité. Ils regardent les passions de l'âme comme autant de maladies, et ils sont dans l'erreur. La religion nous apprend que ces affections, ces différents sentiments que nous éprouvons en nous, viennent de Dieu même; que ce sont autant de moyens qu'il nous fournit pour procurer sa gloire, opérer notre salut; la grâce ne détruit pas les passions, elle les corrige, elle les rectifie, elles tiennent le milieu entre le vice et la vertu, et l'usage seul en fait le mérite ou le défaut. La religion dirige-t-elle l'appétit concupiscible, ce désir du bien qui est en nous ? Lui donne-t-elle Dieu pour objet? Elle communique en même temps la soumission et la confiance, la charité pour le prochain, le mépris des biens de la terre [...]. La colère est un mouvement de l'âme par lequel elle s'élève contre la cause du mal et de l'injure qu'elle ressent, et conçoit le désir de se venger. Cette passion, quand elle a Dieu pour objet, produit le zèle qui réprime les vices, retient les inférieurs dans l'ordre, anime les martyrs ». On ne saurait tenir les passions pour des maux dont le sage peut s'affranchir ou pour des fautes expiables, comme le christianisme l'a parfois soutenu. Aucune passion n'est naturellement mauvaise ; l'affirmer serait offenser le Créateur. Contre les philosophes qui les envisagent comme autant de maladies de l'âme, Valerio reprend la distinction platonicienne du concupiscible et de l'irascible, de la passion qui aveugle et de la passion qui éloigne d'elle-même. Lamy lui fera écho : " on me dira qu'il n'est point permis d'user des moyens aussi injustes que sont les passions [...] les passions sont bonnes en elles-mêmes : leur seul dérèglement est criminel. Ce sont des mouvements dans l'âme, qui la portent au bien, et qui l'éloignent du mal, qui la poussent à acquérir l'un, et qui l'excitent lorsqu'elle est trop paresseuse à fuir l'autre. [...] je ne crois qu'on puisse dire raisonnablement qu'il n'est pas permis de se servir de sa colère pour animer les hommes à chercher le bien qu'on leur pro$\operatorname{pose}^{18}$ ». Selon Bretteville, « la passion n'a été donnée à l'Ame que pour la porter vers le Ciel ; et lui ôter les passions, c'est lui ôter les ailes qui la soutiennent et qui l'élèvent. [...] l'éloquence laisse les passions à l'homme, qu'elle ne peut lui ôter sans le détruire, mais elle sait les régler; elle suit leur pente naturelle mais elle les modère; elle s'accommode à leurs inclinations, mais elle les rectifie [...] la colère devient justice [...] l'envie se tourne en émulation, la jalousie en zèle » (op. cit., p. 319-320). La passion peut faire accéder au bien, à l'amour de Dieu ; la colère est une indignation contre le mal: la doctrine augustinienne de la sainte colère contre le pécheur garde toute sa valeur. Tout comme le directeur de conscience jésuite feint d'accepter la nature pour en extraire les forces menant à la sainteté, laisse libre cours aux passions propres à la nature profane de son pénitent afin, une fois gagnée sa confiance, de l'inciter à s'engager sur la voie de la loi divine, et tire des tendances naturelles ou franchement 
mauvaises l'énergie conduisant au salut, l'orateur chrétien se sert de la nature et des passions comme d'une réalité qu'il est impossible de détruire et dont il faut se rendre maître ${ }^{19}$.

S'il faut choisir entre les types de discours que la tradition rhétorique met à disposition, on optera, dès lors qu'il s'agit de toucher ("en rhétorique, note un auteur, on appelle Passion tout ce qui va au cour »), pour une éloquence forte, mâle et pathétique. Rapin donne en exemple Savonarole et Louis de Grenade qui, à Florence et à Séville, connaissaient l'art d'exciter les passions, possédaient la chaleur nécessaire pour toucher et faisaient trembler leurs auditoires. Botero, Louis de Grenade et jusqu'à Bretteville insistent sur la force de l'éloquence (vis dicendi) plutôt que sur l'art. "Toucher l'auditeur, explique Valerio, c'est exciter en lui des sentiments de plaisir, de douleur, d'amour ou de haine, selon les différents sujets qu'on lui propose. [...] On persuade aisément quand on parle à l'esprit et au cœur par la délicatesse et la beauté des expressions, par la solidité des preuves et la vérité des pensées, quand on enlève son suffrage par une éloquence mâle et majestueuse. »Il indique comment il faut inspirer la terreur et marquer son admiration : " le Prédicateur inspirera des sentiments de terreur s'il exprime avec feu, selon les circonstances, les effets funestes des fléaux publics dont Dieu punit le péché » (le texte latin porte : "terrebit orator si incommota belli, penuriae, pestis, et maximae divinae indignationis effectus ante oculos ponat»). Rollin explique que « ce qui produit les grands et les merveilleux effets de l'éloquence, n'est ni le genre simple et médiocre, ni le genre orné et fleuri ; mais le sublime et le pathétique [...]. Que servirait en effet que l'auditeur fût convaincu de ce qu'on lui dit, et qu'il applaudît à l'éloquence de celui qui parle, s'il n'allait jusqu'à aimer, embrasser, pratiquer les maximes qu'on lui prêche ? [...] C'est en cela que S. Augustin, après Cicéron, fait consister la pleine victoire de l'éloquence. Tout discours qui laisse l'auditeur tranquille, qui ne le remue et ne l'agite point, et qui ne va pas jusqu'à le troubler, l'abattre, le renverser, et vaincre son opiniâtre résistance, quelque beau qu'il paraisse, n'est point un discours véritablement éloquent. Il s'agit de lui inspirer de l'horreur de ses péchés, et de la crainte des jugements de Dieu, de dissiper le charme séducteur qui l'aveugle, et de le forcer d'ouvrir les yeux; de lui faire haïr ce qu'il aimait, et aimer ce qu'il haïssait» (op. cit., p. 498). Certaines figures seront plus particulièrement adaptées au but poursuivi : Louis de Grenade conseille notamment l'exclamation, l'apostrophe, l'hyperbole, l'admiration. Valerio précise que «les grands mouvements, les figures nobles et pathétiques, doivent être répandues dans les différentes parties du discours, l'animer comme le sang répandu dans toutes les parties du corps les anime 


\section{Philippe Roussin}

et leur donne la force et la vigueur ». C'est ainsi, explique Crevier, que la passion peut «se transmettre par votre discours à ceux qui vous écoutent ».

\section{Chaleur, enthousiasme, contagion.}

De la chaleur "nécessaire pour toucher » évoquée par Rapin, Hugh Blair affirmera qu'elle constitue, avec la gravité, la principale caractéristique de l'éloquence de la chaire. Leur union, ajoute l'auteur anglais, forme ce que les Français ont nommé onction, soit " the affective, penetrating, interesting manner, flowing from a strong sensibility of heart in the Preacher to the importance of those truths he delivers, and an earnest desire that they may make full impression on the hearts of his Hearers ${ }^{20} "$ ". Il n'est cependant pas facile d'équilibrer ces deux qualités : "the Warm, when it wants gravity, borders on the theatrical». La manière dont la passion est montrée et se communique par le discours, ce qui est pourtant recherché dès lors qu'il faut échauffer les volontés pour pousser à agir chrétiennement, est bien en même temps ce qui pose problème et fait réfléchir les auteurs. Il est, en effet, identifiés par eux, deux dangers qui menacent la rhétorique chrétienne, sur quoi insisteront ses défenseurs et bientôt ses critiques. Le premier est que le prédicateur, voulant à toute force, pour toucher, montrer qu'il est touché, perde en chemin la finalité de son discours (l'utilité) et, victime de la présomption, ne cherche qu'à plaire, devienne un orateur, se change en un comédien, et transforme la chaire en un théâtre : la manière dont l'orateur chrétien communique sa passion et touche son auditoire est alors le plus souvent décrite en référence à la situation de l'acteur et du spectateur de théâtre. Le second est que sa chaleur même interdise de discerner ce qui distingue le prédicateur d'un frénétique, d'un possédé ou d'un enthousiaste.

Un spirituel comme Louis de Grenade, au dernier quart du XVI" siècle, peut ne voir encore dans cette chaleur du discours qu'un effet de la seule dévotion de l'orateur : "leur principale occupation étant d'élever leur esprit et leur cœur vers Dieu, de s'établir dans une solide et fervente dévotion, et de s'exciter à un très ardent amour des biens célestes et éternels, il arrive que la disposition intérieure de plusieurs d'entre ces personnes est telle, qu'à la moindre étincelle du feu de la Parole de Dieu qui les touche, ils prennent feu comme de la poudre à tirer, et s'enflamment tout d'un coup [...] ainsi les Prédicateurs qui s'appliquent avec ferveur aux choses de Dieu et de l'Esprit, s'embrasent aisément du feu de la dévotion et de la charité, et en échauffent ensuite les cœurs et les esprits de leurs Auditeurs ${ }^{21}$ ". Mais très tôt, dès le premier quart du 
XIII siècle, un auteur jésuite comme G. Mazarini est attentif à prévenir que le prédicateur ne doit pas confondre le don des larmes, marque d'une relation totale à Dieu, avec la faculté de pleurer et le sentimentalisme : «le mot émouvoir se prend en deux façons : premièrement en sa propre signification, qui est de fléchir la volonté à embrasser et faire tout ce que l'entendement lui représente gagné par divers mouvements ; lequel pour en venir à bout, va suscitant en icelle par l'efficace du discours diverses affections proportionnées à la chose qu'on désire persuader. En second lieu, il est pris, mais improprement, pour l'action même de répandre des pleurs. Abus enracinés si avant dans l'âme de l'ignorant populaire qu'il se fait accroire qu'émouvoir n'est autre chose que faire pleurer, et qu'un Prédicateur n'incite point les affections, s'il ne tire les larmes des yeux ${ }^{222}{ }^{\text {». }}$ Que le prédicateur, poursuit-il, se garde d'affecter les larmes et de pleurer lui-même, ce serait faire « acte de bateleur ». Évoquant ces prédicateurs " qui font les passionnés sur tout ", aux gestes trop expressifs et au visage trop comédien, Rapin rappelle que " la chaire ne doit pas être comme le théâtre, où l'on ne va que pour le plaisir : il faut que le prédicateur pense à dire des choses utiles [...]. On ne va au sermon que pour être touché, quand on y va comme l'on doit y aller ${ }^{2.3} »$.

L'autre danger, que glosera surtout la critique rationaliste de la religion, est que l'orateur chrétien, inspiré et emporté par cette forte éloquence, passe pour un frénétique ou un enthousiaste. Souvent, écrit Lamy, les prédicateurs " s'échauffent " et "emploient toutes les plus fortes figures de la Rhétorique "; ils sont, alors, "semblables à un phrénétique qui se sert de son épée pour combattre un ennemi phantastique que son imagination troublée lui fait voir en l'air. Ces Déclamateurs entrent dans des Enthousiasmes, qui leur font perdre l'usage de la raison" (op. cit., p. 181). D'un autre prédicateur, Rapin relève que "sa manière s'était si fort gâtée par les grimaces et les agitations violentes et forcées de tout son corps, qu'on le défendait aux femmes grosses : parce que ses mouvements avaient l'air de convulsions ". Comparant l'éloquence de la chaire en France et en Angleterre, $\mathrm{H}$. Blair note, à la fin du XVII" siècle : " $A$ French Sermon, is for most part a warm animated exhortation; an English one, is a piece of cool instructive reasoning. The French preachers address themselves chiefly to the imagination and the passions; the English almost solely to the understanding. [...] A French Sermon would found in ours ears as a florid, and, often, as an enthusiastic, harangue " (op. cit,. p. 119). En Angleterre, à partir de Locke, de Shaftesbury, plus tard avec Hume, en France, à partir des auteurs anglais et de Bayle, et surtout chez les philosophes des Lumières, la critique de la rhétorique de la chaire comme de ses effets communicationnels et collectifs relèvera toujours davantage des analyses critiques de l'enthousiasme, développées 


\section{Philippe Roussin}

à partir du spectacle des troubles religieux et politiques observés dans la seconde moitié du XVII" siècle, pour conduire au découplage et au clivage du concept que l'on sait : la positivité de l'enthousiasme lorsqu'il s'agit du génie et du domaine de l'art; la négativité dangereuse de l'enthousiasme religieux, "une flamme vive qui gagne de proche en proche, qui se nourrit de son propre feu, et qui loin de s'affaiblir en s'étendant, prend de nouvelles forces à mesure qu'elle se répand et se communique " (art. "Enthousiasme» de l'Encyclopédie).

Les auteurs apprécient le risque de ces deux écueils à l'aulne de ce qui leur paraît être la spécificité de la rhétorique chrétienne : elle se doit d'être populaire; elle s'adresse, à la différence des autres genres de discours, non à un petit nombre de juges mais à une grande assemblée d'individus, qu'elle constitue, en outre, en un spectateur et en un auditeur unique : " vos auditeurs, dit un auteur tardif, ne sont plus à vos yeux qu'un seul individu, qu'un seul être collectif qui les réunit, et les représente tous avec la plus exacte ressemblance. [...] il n'existe, en quelque sorte, par cette fiction oratoire, qu'un seul homme pour l'orateur, dans la multitude des hommes qui l'environne [...] il ne doit parler dans sa composition qu'à un seul auditeur, à un seul infortuné, à un seul coupable. [...] L'orateur tirera même de nouveau avantage d'une grande affluence, où tous les mouvements excités à la fois, comme les vagues d'une mer agitée qui s'entrechoquent de tous les côtés, multiplieront les triomphes de l'art, en formant une espèce d'action et de réaction entre l'orateur et l'auditoire, [...] vous verrez encore, il est vrai dans le temple, des milliers d'auditeurs; mais il n'y aura plus qu'une seule pensée, un seul intérêt, un seul sentiment ${ }^{2+}{ }^{\text {}}$.

A côté de la considération du public uni dans la foi, les auteurs font également réflexion technique sur les raisons, les effets et les mécanismes de la production de l'unanimité de sentiments observable dans ce public, sur la transmission de l'affect de l'orateur à son auditoire. B. Lamy explique les ressorts psychologiques de la communication par la sympathie, au sens de passion simultanée : "Les hommes ne peuvent remarquer que nous sommes touchés, s'ils n'aperçoivent dans nos paroles les marques des émotions de notre âme. Jamais on ne concevra des sentiments de compassion pour une personne dont le visage est riant : il faut avoir des yeux abattus ou baignés de larmes pour causer ce sentiment. Il faut par la même raison que le discours porte les marques des passions que nous ressentons, et que nous voulons communiquer à ceux qui nous écoutent. Les hommes sont liés les uns avec les autres par une merveilleuse sympathie, qui fait que naturellement ils se communiquent leurs passions $[\ldots]$, nous nous revêtons des sentiments et des affections de ceux avec qui nous vivons. [...] C'est un effet merveilleux de la sagesse de Dieu, qui nous a faits premièrement pour lui; et en second lieu les uns pour les 
autres. Car, comme les passions font agir l'âme pour rechercher le bien et éviter le mal, la nature, par cette sympathie, nous porte à combattre le mal qui attaque ceux avec qui nous vivons, et à leur procurer le bien qu'ils souhaitent. Ainsi, puisque nous ne parlons presque jamais que pour communiquer nos affections aussi bien que nos idées, il est évident que pour rendre notre discours efficace, il [...] lui faut donner les caractères de nos affections, qui se communiquent, comme nous venons de le dire, à ceux qui nous entendent parler lorsqu'elles paraissent " (op. cit., p. 141142).

Au contraire, Malebranche, en philosophe, chrétien mais cartésien, critiquant cette source d'erreurs qu'est l'imagination, verra dans la manière dont l'éloquence remue celle des auditeurs un exemple de ce qu'il nommera la "communication contagieuse des imaginations fortes" : "ceux qui imaginent fortement les choses, les expriment avec beaucoup de force, et persuadent tous ceux qui se convainquent plutôt par l'air et par l'impression sensible, que par la force des raisons [...]. Ainsi l'air de leur visage, le ton de leur voix, et le tour de leurs paroles animant leurs expressions, préparent ceux qui les écoutent et qui les regardent, à se rendre attentifs, et à recevoir machinalement l'impression de l'image qui les agite. Car enfin, un homme qui est pénétré de ce qu'il dit, en pénètre ordinairement les autres, un passionné émeut toujours; et quoique sa rhétorique soit souvent irrégulière, elle ne laisse pas d'être très persuasive ${ }^{2.5}$ \%. La rhétorique qui se donne pour but de toucher est ici décrite dans ses effets et ses mécanismes sous l'espèce de la contagion de l'imagination, elle-même, on le sait, produit dérivé, pour Malebranche, de l'imitation des hommes unis entre eux par la ressemblance de nature ${ }^{20}$.

Une telle rhétorique, surtout lorsqu'elle entend mobiliser les passions, devait nécessairement tomber sous le coup de la critique, que celle-ci s'exerce dans le cadre de la controverse et de la polémique religieuses ou de la philosophie politique et morale propre à l'époque de formation de l'État moderne. Alors, la critique de la rhétorique religieuse vise la société chrétienne que l'Église de la Contre-Réforme entend construire, mais aussi le modèle théologico-politique de la communication, via la rhétorique (son recours aux passions, la communication collective des passions religieuses et politiques qu'elle sert). Je me limiterai à en donner deux exemples. Pierre Bayle, comme la majorité des érudits protestants, déclare prendre pour modèle l'éloquence de Sénèque, le sage qui oppose l'impassibilité aux passions. Ciblant la rhétorique de la chaire qu il repère comme formant le fonds de l'écriture de la controverse qui renaît fortement dans les années annonçant la révocation de l'édit de Nantes, il critique en même temps la matière centrale de la Ratio studiorum des collèges jésuites de 


\section{Philippe Roussin}

la Contre-Réforme et l'usage abusif de la parole, vecteur de la violence dans le discours, politique et religieux : " On nous fait apprendre le Latin dans les plus violentes invectives qui se puissent voir, dans les harangues de Cicéron, qui était le plus médisant, le plus emporté et le plus satirique de tous les hommes [...] je ne laisse pas de dire qu'il déchirait trop cruellement ses ennemis, pour être le modèle de l'éloquence Chrétienne. Cependant je ne remarque pas que ceux qui régentent la Rhétorique, nous avertissent de ce grand défaut de Morale qui règne dans les écrits de Cicéron [...] ils nous font employer là toutes les phrases les plus violentes, \& toutes les figures les plus outrées [...] il faudrait [...] nous prémunir contre la contagion des invectives, qui se trouvent dans les anciens auteurs. C'est ce que l'on ne fait pas; au contraire, on les donne à imiter, comme il paraît par le Candidatus Rhetoricae [...]. C'est un Livre [...] qui a grand cours dans les Collèges de la Société, \& qui est tout plein de sanglantes invectives contre Luther et Calvin, proposées aux Écoliers comme un modèle d'amplification, \& un exemple de l'usage qu'il faut faire des figures de la Rhétorique, de l'Exclamation, de l'Apostrophe, de la Prosopopée, etc. et des phrases foudroyantes des Anciens ${ }^{2 ?}$. 》Les critiques que l'homme des Nouvelles de la République des lettres adresse au jésuite Maimbourg, auteur, en 1682, d'une Histoire du calvinisme, visent le prédicateur et le style hyperbolique du " déclamateur qui s'exerce sur les lieux communs de l'invective » et "s'est chargé d'un grand nombre d'épithètes diffamatoires et de descriptions violentes [...] pour faire plus d'impression sur les Lecteurs ${ }^{28}$. Elles se concentrent sur l'homme de l'oral, qui traite ses lecteurs comme s'il parlait à la «foule des ignorants » : "On n'a besoin pour prêcher que de beaucoup d'éloquence, et de quelques pensées vives qui puissent tenir l'Auditeur attentif, et remuer ses passions. Une science superficielle suffit pour cela, lors qu'on ne manque point d'ailleurs d'une belle naissance pour la Chaire. "

Bayle appartient à la tradition philosophique qui voit dans les passions une source de dérèglement du jugement, une source de violence et de menace pour l'ordre social et politique. Cette appartenance explique largement la teneur d'un texte comme la Dissertation sur les libelles diffamatoires, annexé au Dictionnaire historique et critique dès sa première édition, en 1697. Bayle y discute, avant de la réfuter, l'idée reçue qui fait envisager la satire, les libelles, les écrits apologétiques et les textes produits par chaque confession pour les besoins de la controverse, comme un purgatif, un remède pour la communauté, une manière de correction fraternelle. L'Arétin, écrit-il dans l'article du Dictionnaire historique et critique qu'il lui consacre, "se vantait que ses libelles faisaient plus de bien au monde que les sermons»; il disait qu'il fallait " du moins lui accorder quelque gloire pour le service qu'il avait rendu à la vérité, en la 
poussant dans la chambre et dans les oreilles des grands, à la honte de la flatterie et du mensonge [...]. Il ajoute qu'un autre disait : l'Arétin est plus nécessaire à la vie humaine que les prédications, parce que les prédications ne mettent dans le bon chemin que les simples; mais ses Écrits y mettent de grands seigneurs ». Les libellistes et les satiristes, poursuit Bayle, prétendent qu'ils « font du bien à la société, en tant qu'ils empêchent plusieurs personnes de l'un et de l'autre sexe de sortir des bornes de la bienséance; c'est un frein, disent-ils, qui les retient : ôtez-leur la crainte d'être diffamés jusqu'au bout du monde, et dans tous les siècles à venir par quelque satire ingénieuse, il n'y aura point d'excès à quoi ils ne se précipitent. Chansons que tout cela. On ne voit pas que jusqu'ici il $y$ ait jamais eu disette de ces libelles, et cependant le monde n'est point amendé et n'amende point ». Loin d'être un moyen de corriger les mours, libelles et satires sont, en réalité, une peste pour la marche des affaires publiques. Bayle évoque, à leur sujet, la " contagion mortelle de la médisance " (Dissertation sur les libelles diffamatoires) : "l'honneur, la gloire, et la réputation des familles, ces biens mille fois plus précieux que l'or et l'argent, ne tiendraient qu'à un filet, si l'on ne réprimait l'audace et la noire malignité des écrivains satiriques. Ils commenceraient à la vérité par des personnes de mauvaise vie : mais après ce début, ils se répandraient comme la peste, sans aucun discernement sur les lieux saints et les profanes, sur les maisons chastes et celles de prostitution. L'antiquité en aurait fait l'expérience totale, si l'on n'y eut enfin remédié par de bonnes lois, et en soumettant au bras séculier les satiriques, quand on vit que cela passait la raillerie, et quand ceux qui n'avaient pas encore été mordus de ces chiens enragés firent réflexion que leur tour viendrait aussi ; qu'il fallait donc concourir, pour y mettre ordre, avec ceux qui avaient déjà reçu le coup. C'est ainsi qu'en cas d'incendie les voisins ne travaillent pas moins que ceux dont la maison brûle à éteindre l'embrasement ${ }^{29}{ }^{\prime}$. En tant qu'elle entend exciter les passions, fût-ce pour les corriger, cette rhétorique religieuse pose un problème politique. Loin de cimenter la communauté, elle la divise et crée les troubles publics. Les libelles, les satires, la raillerie, l'éloquence enflammée de la chaire sont une peste en cela. Dans le Dictionnaire historique et critique, Savonarole - favorablement cité par Rapin comme l'un de ces prédicateurs possédant la chaleur nécessaire pour toucher - figure, parmi les modèles d'une telle éloquence, l'exemple du débordement de la passion religieuse sur le domaine du politique (Bayle traite de la même manière Pierre Jurieu, le pasteur et inspirateur prophétique du Refuge huguenot de Hollande).

L'image de l'orateur habile à exciter de violentes passions dans son auditoire et, ainsi, à alimenter ou, à la limite, provoquer des séditions et des guerres n'est pas précisément neuve ${ }^{30}$. Chez Hobbes, pour qui le 


\section{Philippe Roussin}

problème central est celui de la constitution de l'État en réponse aux passions et aux mouvements qui font les guerres religieuses et civiles, elle est présente sous l'espèce de cette éloquence qui «émeut les affections de l'âme (comme l'espérance, la crainte, la pitié, la colère) [...] ce qui est d'un merveilleux effet pour le mouvement des passions " et constitue * le vrai caractère de ceux qui excitent la populace aux mouvements » : «ce par quoi ils peuvent mettre en furie leurs auditeurs, dont la tête était déjà mal faite, faire paraître le mal que ceux-ci endurent pire qu'il n'est et le faire imaginer à ceux qui n'en souffrent point du tout : ce par quoi ils peuvent les remplir de belles espérances et aplanir pour eux les précipices, sans aucune apparence de raison, c'est la faculté qu'ils doivent à cette sorte d'éloquence qui ne représente pas les choses telles qu'elles sont et qui, ne se proposant que de déchaîner des tempêtes dans l'âme, fait sembler toutes choses à ceux qui les écoutent, telles qu'elles sont dans le cerveau de celui qui parle, et qui est le premier dans l'agitation ${ }^{31}$.

\section{Sympathie et esthétique.}

Comment parvenir à penser et à analyser ce qui se produit entre les hommes, la communication des sentiments de l'un à l'autre, des uns aux autres, autrement qu'en termes d'influence et de contagion (le contact naturel des créatures dans la hiérarchie du Créateur), autrement dit sans l'idée de Providence, sans le modèle théologico-politique de la communication? Au moyen de la sympathie, comprise à partir des seules lois d'association des idées, répond la philosophie morale anglaise.

Au XVI" siècle, le terme réfère à l'harmonie de l'univers, à une force cosmologique liant ensemble des êtres dissemblables appartenant à des niveaux différents de réalité. On peut trouver trace de cette couche de sens chez Hume, dans le tableau suivant : " faire une revue de l'univers [...] observer la force de la sympathie chez toutes les créatures animées et la facilité de la communication des sentiments d'un être pensant à un autre [...]. Ce désir est encore plus manifeste chez l'homme; celui-ci est la créature de l'univers qui a le désir le plus ardent d'une société [...]. Nous ne pouvons former aucun désir qui ne se réfère pas à la société ${ }^{32}$ ". Mais le terme commence aussi d'être utilisé, à partir du milieu du XVII" siècle, en une autre acception, pour décrire la communication des sentiments d'une personne à l'autre. Le concept tire alors une partie de son sens de l'effort qui est fait pour réfuter les morales de l'égoïsme ${ }^{333}$. Contre les thèses sur l'amour-propre de Hobbes puis de Mandeville, Shaftesbury, Hutcheson, Hume comprennent la sympathie comme une affection naturelle et une source essentielle du plaisir en société : il n'y a pas d'existence 
Des liens humains (toucher, contagion, sympathie)

humaine qui ne soit sociale; la thèse contractualiste est une "pure fiction ", écrit Hume dans le Traité de la nature humaine, comme l'état de nature, observe-t-il, n'est qu'une fiction philosophique. La sympathie sert alors à désigner la dimension immédiatement sociale de toute communauté : " nulle qualité de la nature humaine n'est plus remarquable, à la fois en elle-même et dans ses conséquences, que la tendance naturelle que nous avons à sympathiser avec les autres et à recevoir par communication leurs inclinations et leurs sentiments $(T N H$, p. 417).

Cette sympathie est dans notre nature : elle s'explique d'abord par la ressemblance, les liens du sang, la contiguïté. Son existence permet de rendre compte de toute une série de phénomènes sociaux, l'amour des parents, le caractère national ou la contagion du rire : " il est manifeste que la nature a conservé une grande ressemblance entre toutes les créatures humaines et que nous n'observons jamais en autrui de passion ni de principe dont nous ne puissions trouver, à un degré ou à un autre, l'analogue en nous. [...] cette ressemblance doit extrêmement contribuer à nous faire entrer dans les sentiments d'autrui et à nous les faire embrasser avec facilité et plaisir. Ainsi trouvons-nous que, lorsqu'il y a, outre la ressemblance générale de nos natures, une similitude partielle de mours, de caractère, de pays ou de langage, la sympathie s'en trouve facilitée » $(T N H, p$. 419). Naturellement, j'éprouve de la sympathie pour ceux qui me ressemblent : selon les circonstances, mes proches, mes pareils, mes parents. Cette sympathie " s'étend rarement au-delà des amis, et de la famille, ou, au plus, au-delà du pays natal »; c'est dire si la générosité humaine est naturellement limitée. Hume la décrit en termes psychologiques, comme une communication de sentiments d'une personne à une autre, involontairc et mécanique, ne reposant sur aucune action consciente et intentionnelle; il lui arrive alors d'employer indifféremment, pour la nommer, les termes de « sympathie » ou de " contagion». Elle désigne la tendance qu'a l'individu à adopter, à " attraper » les sentiments et les croyances des autres : "les passions sont si contagieuses qu'elles passent avec la plus grande facilité d'une personne à une autre et qu'elles produisent des mouvements correspondants dans tous les cœurs humains" ( $T N H, p$. 732). Il y a, dans cette description, un renversement qui change la manière de concevoir les passions et - on le verra - les fictions : leur vivacité plus ou moins grande est exclusivement le fait des relations de proximité, de ressemblance, bref, de nos associations d'idées et de notre imagination.

Limitée aux parents, aux proches, aux amis, aux coreligionnaires, la sympathie naturelle ne fait que consacrer des ressemblances et des liens déjà existants. Sa limite, considérée en termes politiques et religieux, consiste dans sa partialité. Selon les circonstances, elle renforcera les divisions et pourra contribuer à enflammer l'esprit de faction : "la sédi- 


\section{Philippe Roussin}

tion populaire, le zèle partisan, une obéissance aveugle à des chefs de faction, voilà quelques-uns des effets les plus évidents, quoique les moins louables, de cette sympathie sociale présente dans la nature humaine» (Enquête sur les principes de la morale). Hume rejoint ici la description de la contagion laissée par Shaftesbury dans sa Lettre sur l'enthousiasme (1708): «on peut légitimement traiter toute passion de panique, lorsqu'elle s'excite dans une multitude, et qu'elle se propage par la vue ou, pour ainsi dire, par un contact de sympathie [one may with good reason call every passion panic which is raised in a multitude and conveyed by aspect or, as it were, by contact or sympathy]. C'est ainsi qu'on peut appeler panique la fureur du peuple, lorsque la rage se porte à l'excès comme nous l'avons vu quelquefois, et surtout quand la religion y entre. Dans cet état tout, jusqu'à son aspect, est contagieux. La fureur passe successivement sur tous les visages, et on gagne le mal aussitôt qu'on l'aperçoit ${ }^{3+}$ ".

La sympathie ne se confond cependant pas avec sa forme restreinte; elle est potentiellement autre chose que cette relation et cette émotion passives, cette empathie, la communication mécanique et involontaire de sentiments et d'affects d'un sujet à un autre, susceptible de produire l'émotion contagieuse et le zèle religieux qui se propage. Ou plutôt, dans la mesure où les passions sont les seules causes qui puissent être assignées aux actions des hommes, sa partialité ne peut être corrigée que si la sympathie peut s'étendre, pour devenir un des ressorts du jugement moral. Bien qu'elle serve à réfuter les passions égoïstes de Hobbes, elle n'est pas, en effet, un principe d'altruisme : «il n'est pas d'humain, ni certes de créature sensible dont le bonheur et le malheur ne nous touchent en quelque mesure, quand ils sont proches de nous et qu'on les représente sous de vives couleurs : mais cela procède uniquement de la sympathie et ce n'est pas une preuve de la réalité d'une telle affection universelle pour l'humanité » (TNH, p. 597-598). Le propos consiste à la comprendre à partir des opérations de l'esprit (ressemblance, contiguïté et causalité), à l'étendre pour en faire une affection sociale, partant, à la libérer de ses formes restreintes, en défaisant sa forme limitée, partiale, dangereuse, dans une sympathie réflexive : "le problème de la société en ce sens, commente Deleuze, n'est pas un problème de limitation, mais d'intégration. Intégrer les sympathies, c'est faire que la sympathie dépasse sa contradiction, sa partialité naturelle. [...] le problème moral et social consiste à passer des sympathies réelles qui s'excluent à un tout réel qui inclut les sympathies elles-mêmes ${ }^{3.3}$ ". La sympathie, analyse Hume, est toujours fonction de la contiguïté et des liens de causalité. Ses degrés varient; sa force est fonction de l'ensemble des autres rapports que le 
sujet est susceptible, selon les circonstances, d'entretenir avec son objet. Elle est donc plus ou moins étendue selon que l'objet auquel elle se rapporte est susceptible d'avoir des rapports plus ou moins grands avec le sujet. En l'occurrence, « nous sympathisons avec des personnes proches de nous plus qu'avec des personnes éloignées de nous, avec nos connaissances plus qu'avec des étrangers » $(T N H$, p. 706). Plus l'objet nous est proche, plus la sympathie naturelle à son endroit sera forte; plus l'objet est lointain et moins elle s'exercera : "l'imagination ne peut oublier totalement les points de l'espace et du temps où nous existons effectivement. [...] tout objet qui nous est contigu dans l'espace ou dans le temps, se conçoit avec une force et une vivacité particulières et l'emporte sur tout autre objet dans son influence sur l'imagination. [...] des objets proches doivent avoir une influence de beaucoup supérieure à celle d'objets distants et éloignés. En conséquence, nous trouvons dans la vie courante que les hommes s'intéressent principalement aux objets qui ne sont pas très éloignés dans l'espace et dans le temps» (TNH, p. 537-543). La sympathie se rapporte ainsi à autrui selon des degrés de proximité ou d'éloignement qui la rendent plus ou moins vive : "les sentiments d'autrui ont peu d'influence si autrui est loin de nous, et ils réclament la relation de contiguïté pour se communiquer entièrement. Les relations par le sang, qui sont une espèce de causalité, peuvent parfois contribuer à produire le même effet [...]. Toutes ces relations, quand elles s'unissent ensemble, transportent l'impression et la conscience de notre propre personne à l'idée des sentiments ou des passions d'autrui et nous les font concevoir de la manière la plus vive et la plus forte » (TNH, p. 419-420).

Pour devenir un ressort du jugement et du monde moral, la sympathie - le fait d'être ému par le malheur des autres - doit ne pas se limiter au moment présent et au proche, et pouvoir s'exercer hors de ses limites naturelles, comme par-delà les variations de proximité ou de distance. La variation quotidiennement éprouvée de ma propre situation par rapport aux objets et aux personnes (un homme distant de nous peut devenir en peu de temps une connaissance intime) $m$ 'apporte la preuve de la relativité de ma situation et de mon point de vue particuliers. Plus que cette expérience de la continuelle mobilité de notre situation, c'est, cependant, la correction introduite par le jugement par rapport à l'inégalité des émotions ou à la variation de la sympathie qui permet le jugement moral, rendant par là «nos sentiments plus publics et plus sociaux » : «la communication des sentiments dans la société et la conversation nous fait donc former un critère général et immuable qui nous permet d'approuver ou de désapprouver les caractères et les mœurs. Bien que le cœur ne seconde pas toujours ces notions générales, ou qu'il ne règle pas sur elles son amour ou sa haine, pourtant elles suffisent pour la conversation et 


\section{Philippe Roussin}

elles servent tous nos desseins en société, en chaire, au théâtre et dans les écoles » $(T N H$, p. 731). Le correctif du jugement résout la contradiction entre les limites de la générosité et l'étendue de la sympathie, et permet d'intégrer les passions que la sympathie naturelle ne dépasse pas, limitée qu'elle est par les catégories de la famille, de l'amitié, du voisinage, du parti ou de la confession.

L'exercice correctif de la raison correspond, dès lors, avec le point de vue d'un spectateur. Celui-ci n'est plus affecté de l'extérieur, absorbé par une émotion; il devient un agent moral capable de "faire abstraction de sa situation privée et particulière » et de choisir "un point de vue que d'autres puissent partager avec lui »: «il doit mettre en jeu un principe universel de la nature humaine et toucher une corde susceptible de faire vibrer toute l'humanité en accord et en harmonie [he must move some universal principle of the human frame, and touch a string to which all mankind have an accord and symphony] " (Enquête sur les principes de la morale) ${ }^{30}$. Loin d'être transmise par les seuls signes extérieurs au moyen desquels autrui cherche à la communiquer, comme dans la rhétorique chrétienne, l'affection de sympathie devient une construction de l'esprit. Entrer dans les sentiments d'autrui n'implique plus que nous nous perdions nous-mêmes, comme dans la sympathie émotionnelle, où l'identité personnelle disparaît dans l'unanimité des sentiments, où la différence du soi et de l'autre se perd dans la communication contagieuse des affections : la sympathie pour autrui suppose, au contraire, la conscience certaine de nos propres sentiments et de nos propres croyances. L'introduction d'une réflexivité autorise la réversibilité des rôles du spectateur et de l'acteur, interdite dans la forme restreinte et unidirectionnelle de la sympathie naturelle qu'est la contagion.

Ma dernière série de remarques concernera l'importance de la réflexion morale de Hume pour la position esthétique qu'il a prise dans le débat sur la fiction, le théâtre et notamment le paradoxe tragique (comment le spectacle des passions en elles-mêmes désagréables et noires peut-il nous réjouir?), l'un des plus longs et des plus embrouillés du xilll siècle. Alors même que l'enthousiasme religieux faisait l'objet d'une critique rationaliste serrée sous l'espèce de la contagion, toucher, émouvoir, s'enthousiasmer, avons-nous dit, ont poursuivi leur destin et envahi le champ de la rhétorique puis de l'esthétique. Toucher est même devenu et resté une sorte d'idéal esthétique, aussi longtemps que l'art et l'œuvre d'art ont été essentiellement envisagés comme le produit de l'enthousiasme du génie et, du côté du récepteur (spectateur ou lecteur), des émotions et des passions. L'abbé Dubos - dans ses Réflexions critiques sur la poésie et la peinture (1719), auxquelles se réfèrent tous les auteurs du siècle et dont 
Des liens humains (toucher, contagion, sympathie)

Hume réfute les thèses en même temps que celles de Fontenelle dans of Tragedy - puis le sentimentalisme anglais mettent au premier plan, lorsqu'ils traitent du théâtre, de la tragédie et de l'art, la capacité qu'a ce dernier de toucher le cœur et de remuer l'âme. La tragédie n'a pour but que de purger les passions, d'exciter la terreur et la compassion. L'abbé Dubos, cela a souvent été dit, apprécie le contenu esthétique de l'œuvre en fonction de l'effet que cette dernière produit sur le spectateur - sa théorie est tout entière orientée vers celui-ci : "le sublime de la poésie et de la peinture est de toucher et de plaire, comme celui de l'éloquence est de persuader. Il ne suffit pas que vos vers soient beaux [...] il faut encore que ces vers puissent remuer les cœurs et qu'ils soient capables d'y faire naître les sentiments qu'ils prétendent exciter. [...] Soyez toujours pathétiques [...], ne laissez jamais languir vos spectateurs, ni vos auditeurs. [...] Si cet enthousiasme divin, qui rend les peintres poètes, et les poètes peintres manque à nos artisans, s'ils n'ont pas, comme le dit M. Perrault, Ce feu, cette divine flamme, l'esprit de notre esprit et l'âme de notre âme [...], les uns et les autres restent toute leur vie de vils ouvriers et des manœuvres ${ }^{37}$ ». Sa théorie de l'art, comme l'a analysé Cassirer, limite au seul sentiment le contenu esthétique de l'œuvre et restreint le sentiment lui-même à la seule émotion, l'intensité de l'excitation et de l'émotion que l'œuvre produit en nous devenant l'unique mesure de sa valeur esthétique. Au demeurant, l'émotion ressentie n'a, en elle-même, rien de particulièrement esthétique : c'est, dit Dubos, une "émotion naturelle, qui s'excite en nous machinalement, quand nous voyons nos semblables dans le danger ou dans le malheur ", qui "n'a d'autre attrait que celui d'être une passion dont les mouvements remuent l'âme et la tiennent occupée $»$. Dubos considère indifféremment l'émotion du spectateur des combats de gladiateurs, de l'exécution publique d'un criminel ou d'une tragédie : " on va voir en foule un spectacle des plus affreux que les hommes puissent regarder; je veux dire le supplice d'un autre homme qui subit la rigueur des lois sur un échafaud [...] le monde dans tous les pays va voir en foule les spectacles horribles dont je viens de parler [...] les Romains sentaient à l'amphithéâtre une émotion qu'ils ne trouvaient pas au cirque ni au théâtre. Les combats des gladiateurs ne cessèrent à Rome qu'après que la religion chrétienne y fut devenue la religion dominante ».

La comparaison entre le spectacle tragique et le spectacle d'un supplice se rencontre également chez Burke quand il entend prouver que l'art de la poésie et de l'éloquence n'est ni l'imitation ni la fiction, mais consiste à " frapper l'âme de l'orateur et des auditeurs par l'effet des choses ", « à émouvoir plutôt par la sympathie que par l'imitation». Plus le spectacle de la tragédie approche de la réalité " et éloigne de nous toute idée de fiction, plus son pouvoir est parfait. Mais de quelque genre que soit ce 


\section{Philippe Roussin}

pouvoir, il n'atteindra jamais l'effet que produirait dans la réalité l'action imitée. Qu'on choisisse un jour pour représenter la plus sublime et la plus touchante de nos tragédies [...] et quand la salle sera remplie de spectateurs, au moment que leurs âmes seront comme suspendues dans l'attente, qu'on vienne annoncer qu'un criminel d'état du premier rang est sur le point d'être exécuté dans la place voisine; à l'instant la solitude de la place prouvera la faiblesse comparative des arts d'imitation, et attestera la puissance irrésistible de la sympathie réelle ${ }^{38}$.

Tenant de l'imitation, l'abbé Dubos soutient, pour sa part, que si nous pouvons prendre plaisir à des spectacles affligeants, c'est que la passion représentée fait une impression moindre que l'objet qu'elle imite : la copie de l'objet (l'œuvre) excite en nous une copie de la passion que l'objet y aurait excitée. Un tableau et une tragédie nous émeuvent et nous touchent ainsi "sans nous affliger réellement ». Fontenelle, dans ses Réflexions sur la poétique, met, lui, le plaisir sur le compte de l'irréalité du spectacle, dont le spectateur a conscience.

La position de Hume est tout autre. Elle le conduit à rechercher une source, essentiellement intrinsèque et autonome, au plaisir du spectacle tragique, donc à refuser que les effets de la représentation théâtrale puissent être exclusivement envisagés à partir des états émotifs qu'elle engendre et à considérer plutôt les différences de forme que prend la sympathie dans la réalité et dans les arts. S'il donne à plusieurs reprises la situation du spectateur de théâtre comme exemple de l'existence de la sympathie sociale, il ne fait pas de la réaction du spectateur au drame qu'on lui propose une réaction de sympathie pour les malheurs représentés sur la scène : la tragédie ne peut être réduite à un moyen de faire naître la pitié. Au lieu de penser le théâtre à partir de la seule expérience émotionnelle du spectateur, comme une sympathie face au spectacle de malheurs, Hume explique que la cause du plaisir est à rechercher dans la présence de l'artefact et dans les traits de la fiction. Dans Of Tragedy, où il réfute successivement Dubos et Fontenelle, il rend ainsi compte du "plaisir inexplicable éprouvé au spectacle de la douleur, de la terreur, de l'anxiété et des autres passions qui sont en elles-mêmes désagréables " et mettent les spectateurs mal à l'aise ("plus ils en sont touchés et affectés, plus ils sont ravis du spectacle ") : "Il est certain que la même scène de détresse qui nous plaît dans une tragédie nous procurerait, si elle se passait réellement là, sous nos yeux, le malaise le moins feint. [...] cet effet extraordinaire procède de l'éloquence même avec laquelle la scène désolante est évoquée [...] l'art consommé pour en rassembler tous les détails pathétiques, et le jugement exercé pour les exposer dans l'ordre : l'exercice, dis-je, de ces nobles talents, en même temps que la force de l'expression et que la beauté des rythmes oratoires diffusent la plus grande satisfaction dans 
l'auditoire, et suscitent les plus délicieux plaisirs. Par ce moyen, non seulement, le tourment des passions tristes se trouve maîtrisé et effacé par l'action de quelque chose de plus fort et d'une essence opposée, mais aussi l'élan d'ensemble de ces passions se trouve transformé en plaisir et vient augmenter le délice que l'éloquence soulève en nous [...]. Cette particularité a pour effet d'adoucir encore davantage les mouvements de la passion et de convertir intégralement le sentiment en un plaisir puissant et régulier [...]. C'est ainsi que la fiction de la tragédie adoucit la passion, par l'infusion d'un nouveau sentiment et non pas simplement par l'affaiblissement et l'atténuation de la peine ${ }^{34}$. $》$

Il n'y a pas qu'une différence de degré entre l'œuvre d'art et les passions réelles, mais une différence de nature; l'œuvre n'est pas seulement un moyen d'exciter la pitié, de faire effet sur le spectateur. Si elle plaît, ce n'est pas dans la mesure où les passions qu'elle met en scène seraient plus faibles (Dubos) que les passions de la réalité ou plus proches (Burke) d'elles. L'objet d'art a un mode d'existence qui lui est propre, qui ne passe pas par son rapport à l'objet réel : "l'imagination a son jeu de passions qui lui appartient, et dont dépendent grandement nos sentiments de beauté. Ces passions sont mues par des degrés de vie et de force, qui [...] ne dépendent pas de l'existence réelle de leurs objets» $(T N H, \mathrm{p} .711)$. La tragédie ne consiste pas dans le transport des affects des acteurs au spectateur et au public unanime. La fiction est autonome, elle est une entreprise inventée, un artefact, un rapport de l'homme à son imagination; elle n'est pas une copie, l'art n'est pas, comme l'écrit Dubos, une vie empruntée, feinte ou mensonge; elle est un produit spécifiquement humain de l'imagination libre, elle-même positivement considérée. La tragédie ne copie ni ne se borne à transmettre les affects existants, fussentils adoucis; elle fournit en passions l'imagination des spectateurs, les irréalise et les transforme en fictions. Dans l'Enquête sur l'entendement humain, détaillant les mécanismes de l'association d'idées (avant de donner, dans la section $\mathrm{V}$, la fiction et son infinie variété comme exemples de la liberté de l'imagination), Hume prend comme modèle du rapport de cause à effet la manière dont les récits et les compositions narratives construisent l'enchaînement des événements. En poéticien, il met, de même, l'accent sur l'unité de la fable, la connexion des événements entre eux. Sans elles, ajoute-t-il, "l'imagination du lecteur, enflammée par une telle suite de descriptions poétiques, et ses passions, agitées par une continuelle sympathie avec les acteurs, retomberaient nécessairement longtemps avant le terme du récit et nécessairement sombreraient dans la lassitude et le dégoût par suite de la violence répétée des mêmes mouvements ».

De même que la communication des sentiments entre les hommes peut 


\title{
Philippe Roussin
}

être décrite - et vécue par un spectateur impartial - non plus en termes de contagion mais de sympathie, il faut un nouveau découpage, un nouvel équilibre entre passions et imagination, comme aussi la reconnaissance par l'empirisme de la fiction comme produit de l'imagination et artefact, pour que commencent à pouvoir être distinguées émotion et expérience esthétique, quitte, dès lors que l'enquête montre que le jugement esthétique fait rarement l'objet d'une unanimité, à s'interroger sur la nature du goût et sa diversité.

Philippe Rol sistix

centre de recherehes sur less arts el le langage

\author{
volis
}

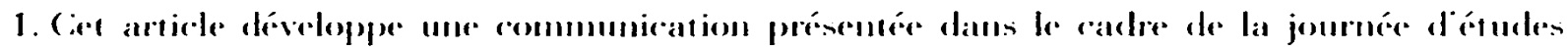

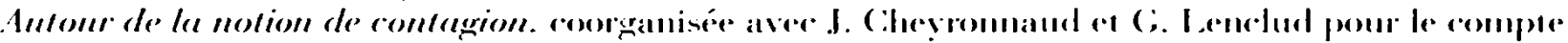

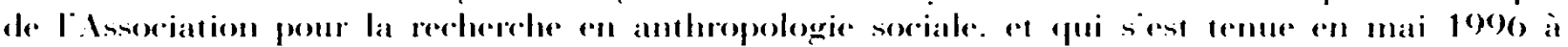
lithest. à llarseille.

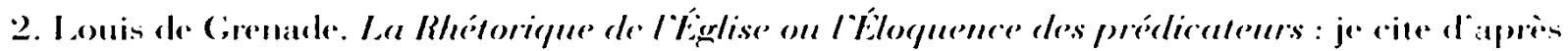
la traduction française de N. Bines. Partis. 1698. livere I. Vil. p. 68-69.

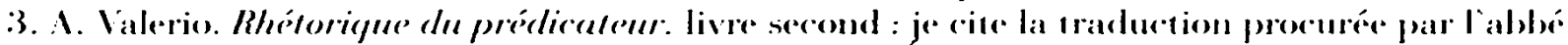

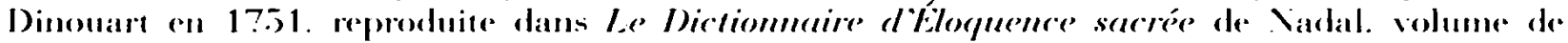
ta . Vourelle Lincucloprédie théologique de Vigne. Paris. 1851.

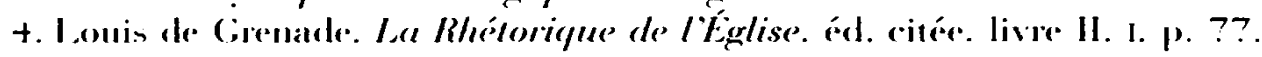

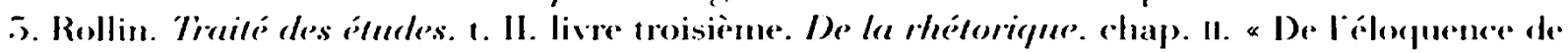
lat chater क. id. de 1720. p. to1.

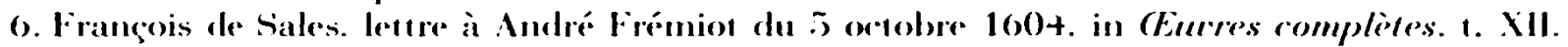
id. d'Annecy: 1902. p. 304.

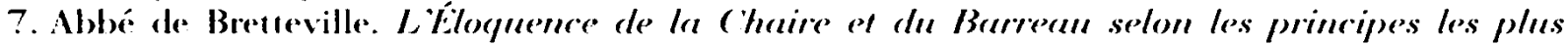

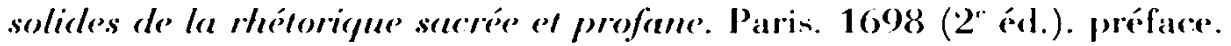

8. B. I any: De Lart de parler (16?2) : éd. citée: La Rhétorique ou liAt de parler (1715). p. $: 37: 3-37+4$.

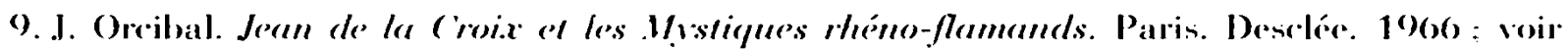

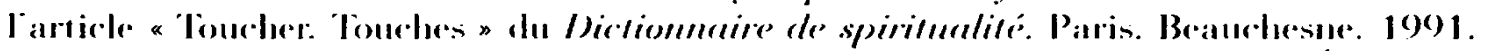

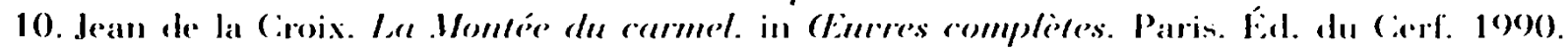
p. $10 . \overline{5}+10 . \overline{5} . \overline{3}$.

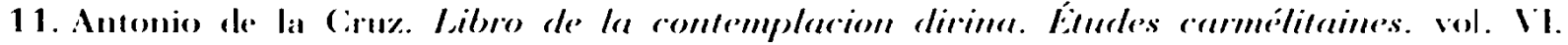
avril 1933.3. p. $271 \mathrm{~s} \%$

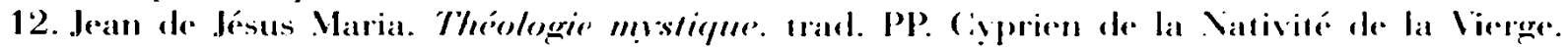
Parris. 16.50. p. 217-218.

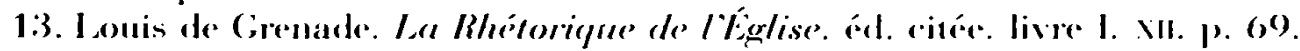

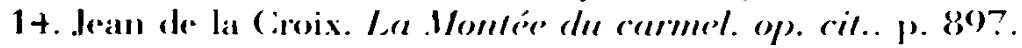

1.). Rollin. Trailé des éfucles. op. ril. p. +o9.

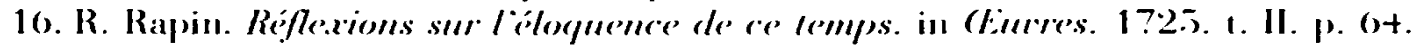

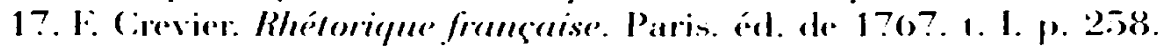

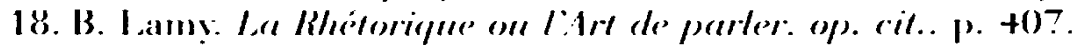




\section{Des liens humains (toucher, contagion, sympathie)}

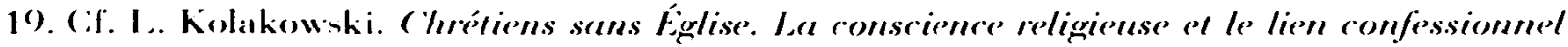
aul dix-septieme sierde. Partis. (Gallimard. 1969.

20. II. Blair. Ierfures on Rhetoric anerl Belles Lethes (178:3). H. F. Harding (ed.). Carbondale. Southern Illinois l niversity Press. 1965. vol. I. lect. XxIX (sur l'áloguence de la chaire). p. 107. Blair est écossais. ministre protestant. auteur de sermons el professecur de rhétorique.

21. I. de Grenade. La Rhétorique de IEgglise. éd. citée. livere I. XII. p. 70-?1.

22. (;. Mazarini. Pratica brere del predicare. Ventise. 1615: je cite d'apres la traduction fran-

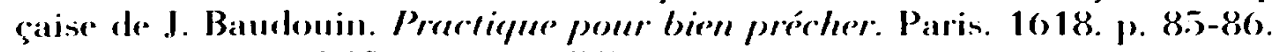

23. R. Rapin. Réflextoms sur l'éloquence de co lemps. op. ril.. p. 7:3-?t.

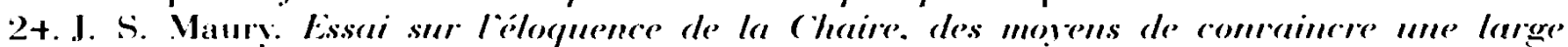

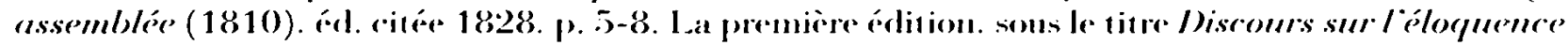
de la chaire. ent de $177 ?$ ?

25. Malebranche. De la Recherefhe de la cérité. in CEuress. Paris. Gallimard. coll. "Bibliothègue de la Pleiade ». 19?9. p. 2.50-251. Malebranche songe en particulier atux visionnaires et aux esprits forts : "les imaginations forles despluelles il fatut eviter aver soin limpression et la contagion. sont ertains esprits par le monde. qui affectent la qualité desprits forts"s.

20. Que la contagion. qui est un mal. puisse eonstituer un effet de l'imitation. qui est un bient.

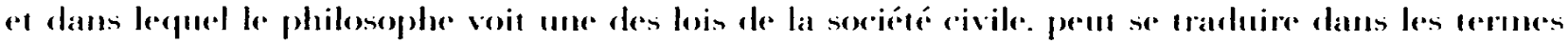
de la thétorigue. Celle-ci doit procurer des exemples à imiter. Pour Fénelon. les panégyristes

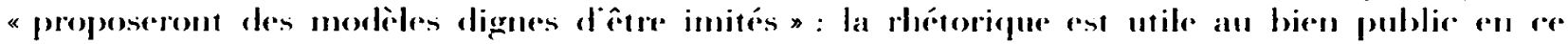
quille est formatriere de modeless : il \& ne faut louer un heros. que pour apprendre ses vertus aut

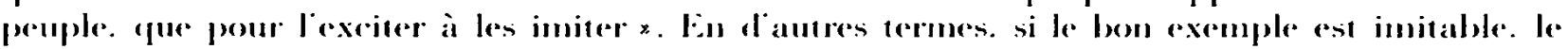
matuais. lui. est contagieux : \& les fautes des grands hommes. érit en ce sells Cerevier: sont contagieuses \$. 1, mot se charge d'une valeur inévitablement polemique che\% les bons auteurs à loreasion de la controverse religieuse ou de la lutre antiphilosophique. Il est très présent dans le

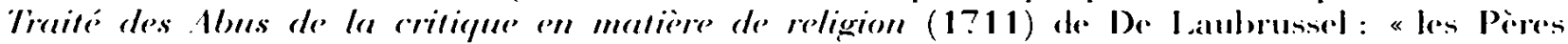
comprenaient bien quarant cous hérite d Mdam lenvie de toucher à l'arbe défendu de la seienee

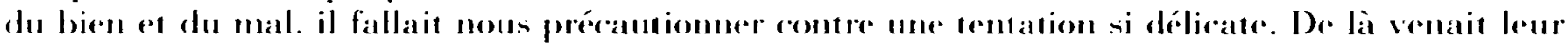

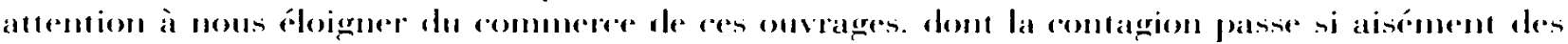

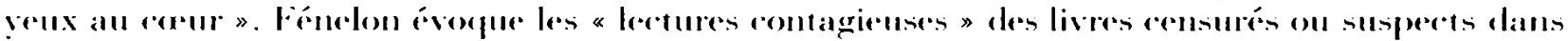

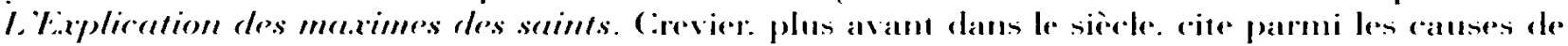

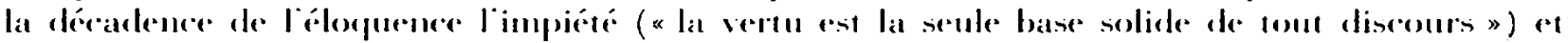

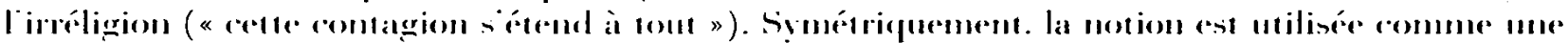

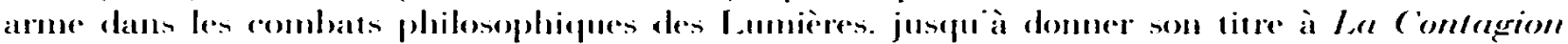

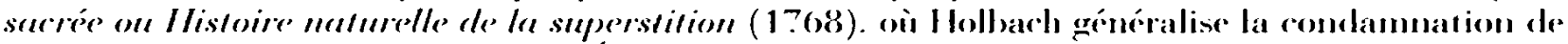
lenthousiasme à la religrion el allx Fyglises : les extases. les visions el les inspirations den haut

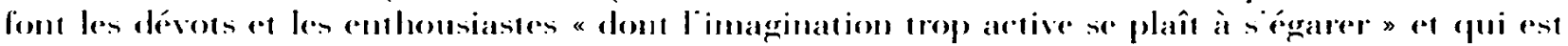

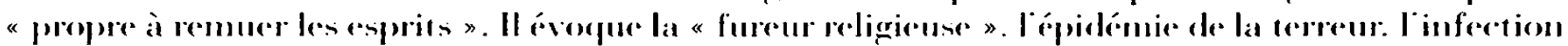
du venin de la superstition et les prêtres qui " ne peurent qu equarer limagination. allumer la déraison. "r faire agir les peruples en insonsés".

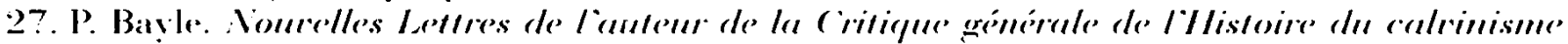

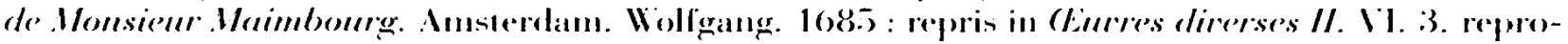

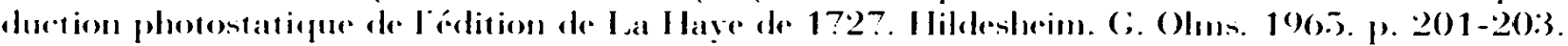

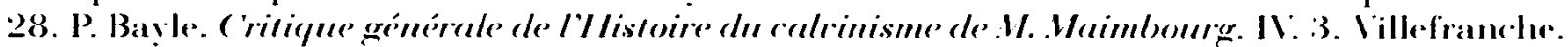
1082. in CEurres direrses II. op. rit.

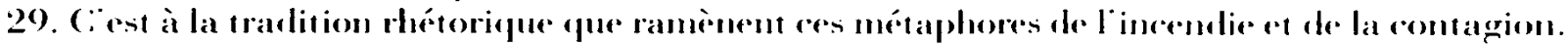
Le discours passionmé porte en lui la capacite denflammer les juges (incenderns judicess) : less

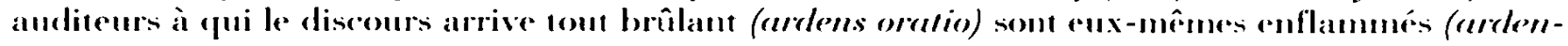

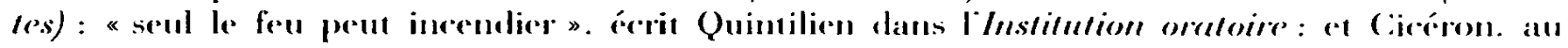
live II du be oratore: * il niest pas de matière si inflammable gu elle soit. qui sembrase saths quion y mette le feut : de même il 11 y a point dêne tellement disposée à recevoir les impressions de l'orateur: qu elle puisse sallumere pour ainsi dire. si l'on napproche de elle la flamme dont on est soi-même dévoré ". I ees libelles somt l'étincelle qui met le feu à toute la plaine.

30. (ff. "Passions et politique". Roe Descartes. n" 12-13. Paris. Alloin Michel. 1905. en par-

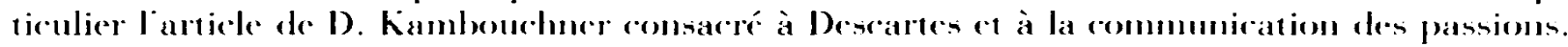




\section{Philippe Roussin}

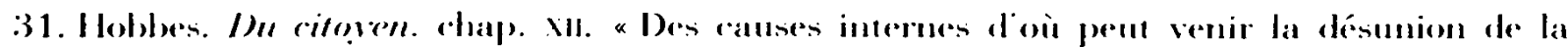
société civile ". Parin. Le livere de Poche. 1906. p. 197-198.

32. Hume. Truite de la mature hummine (17:39). Paris. Aubier-Montaigne. 1908. 1. 11. p. +is? (dorénavant alorége en $T 2 \mathrm{~N} / \mathrm{l}$ ).

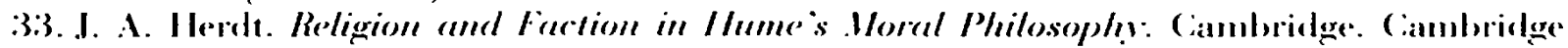
liniversit! Press. 1907.

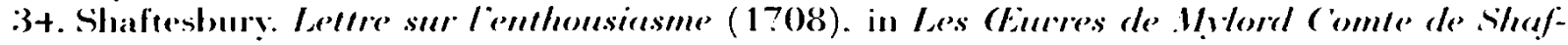

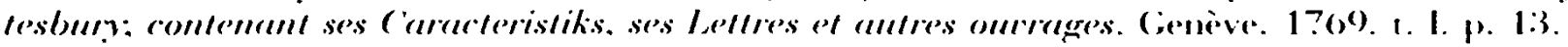

35. (: Deleuze. Empirisme el Sieljectirile. Paris. Pl F. 1953. p. 20-27.

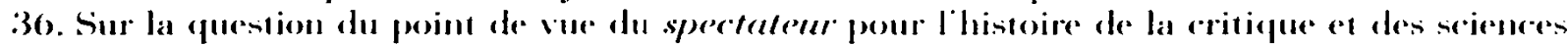

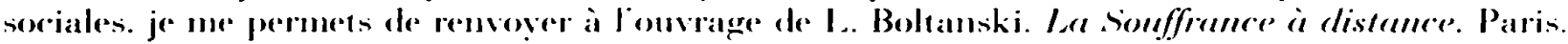
Métailie. 100:3.

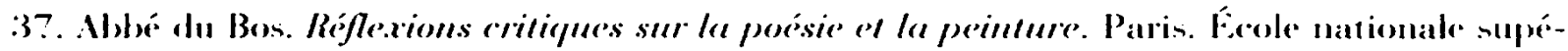
rieure des beaux-arts. 1993. p. 171-172.

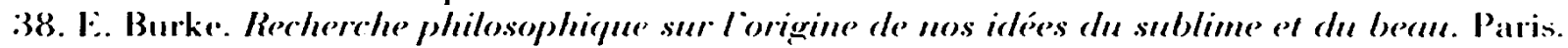
virit. 19?:3. p. 308 e $8+-85$.

39. Hume. "De la tragédie " in Essatis esthétiques. Paris. Vrin. 197+. 1. Il. p. 10+-11+: 1elle est également la conchusion des réflexions d'Adam Sinith sur les arts d'imitation : l'émotion esthé-

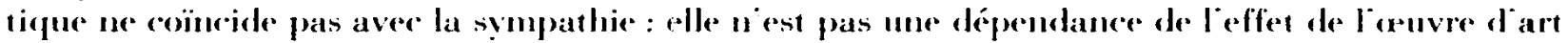

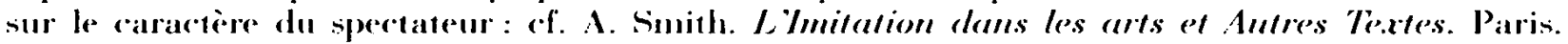
Vine 199? preface de D. Deleule. 